\title{
Analysis of the mechanisms underlying windup using a detailed biophysical model of WDR neurons
}

\author{
Paulo Aguiarr ${ }^{1,2^{*}}$, Mafalda Sousa², Deolinda Lima ${ }^{2,3}$ \\ From Nineteenth Annual Computational Neuroscience Meeting: CNS*2010 \\ San Antonio, TX, USA. 24-30 July 2010
}

Windup is characterized as a frequency-dependent increase in the number of evoked action potentials in dorsal horn neurons, in response to electrical stimulation of afferent $\mathrm{C}$-fibers. This phenomenon was first described in the mid-sixties but the core mechanisms behind it still remain elusive. Several factors affecting its dynamics have been identified but the distinction between modulating mechanisms from generating mechanisms is not always clear. Several mechanisms contribute to the excitation of dorsal horn neurons exhibiting windup and one of our main aims was to help making this distinction. The approach presented here relies on mathematical and computational analysis to study the mechanism(s) underlying windup. To our knowledge, this is the first biophysical model addressing the windup mechanism, and one of the few existing models for information processing in the spinal cord' dorsal horn. From experimentally obtained windup profiles, we extract the time-scale of the facilitation mechanisms which are capable of supporting the characteristics of windup. Guided by these values and using simulations of a biologically realistic compartmental model of a wide dynamic range (WDR) neuron, we are able to assess the contribution of each mechanism for the generation of action potentials windup. We show that the key mechanisms giving rise to windup is the temporal summation of AMPA and NMDA long-lasting post-synaptic responses taking place on top of a membrane potential cumulative depolarization. Calcium activated non-specific cationic currents driven by calcium influx from L-type calcium channels and synaptic currents support this cumulative depolarization and plateau formation in WDR neuron membrane potential. The effects of different non-homogeneous stimulation protocols are explored and their important role in clarifying many aspects of the windup generation is demonstrated. The models were constructed in the well established simulation environment NEURON and are used to produce several predictions which can be tested experimentally.

\section{Acknowledgements}

The authors would to thank Pascal Fossat and Frédéric Nagy for providing the nociceptive flexion reflex data used in the regression analysis. The first author was supported by the Centro de Matemática da Universidade do Porto, financed by FCT through the programmes POCTI and POSI, with Portuguese and European Community structural funds, and by a FCT Grant PTDC/SAU-NEU/68929/2006.

\section{Author details}

${ }^{1}$ CMUP - Centro de Matemática da Universidade do Porto, Porto, Portugal. ${ }^{2}$ IBMC - Instituto de Biologia Molecular e Celular, Porto, Portugal.

${ }^{3}$ Faculdade de Medicina da Universidade do Porto, Portugal.

Published: 20 July 2010

doi:10.1186/1471-2202-11-S1-014

Cite this article as: Aguiar et al: Analysis of the mechanisms underlying windup using a detailed biophysical model of WDR neurons. BMC Neuroscience 2010 11(Suppl 1):014.

* Correspondence: pauloaguiar@fc.up.pt

'CMUP - Centro de Matemática da Universidade do Porto, Porto, Portugal 